\title{
ESTUDO DAS ISOTERMAS DE DESSORÇÃO DE SEMENTES DE CEVADILHA VACARIANA (Bromus auleticus TRINIUS)
}

\author{
L.C. de OLIVEIRA ${ }^{1}$, C.G. FLORES ${ }^{2}$, J.C.P. OLIVEIRA ${ }^{3}$, A.R.F. ALMEIDA Al, $^{1, *}$
}

${ }^{1}$ Universidade Federal do Pampa, Programa de Pós-Graduação em Engenharia, Campus Alegrete e Bagé

${ }^{2}$ Universidade Federal do Pampa, Curso de Engenharia Química, Campus Bagé

${ }^{3}$ Empresa Brasileira de Pesquisa Agropecuária, Embrapa Pecuária Sul

*e-mail para contato: andre.almeida@unipampa.edu.br

RESUMO - Visando uma futura análise do processo de secagem, o presente trabalho teve como objetivo estudar as isotermas de dessorção das sementes de cevadilha vacariana (Bromus auleticus Trinius) nas temperaturas de 40, 50 e $60^{\circ} \mathrm{C}$. Os experimentos foram desenvolvidos a partir do método gravimétrico estático em estufa de circulação de ar forçada com onze diferentes concentrações de ácido sulfúrico, garantindo uma variação da umidade relativa do ar na faixa de 5 a $89 \%$. Os dados experimentais foram ajustados aos modelos matemáticos de BET, GAB, Oswin, Peleg e Henderson. Os resultados obtidos mostraram que os valores da umidade de equilíbrio possuem uma forte dependência com a atmosfera e com a temperatura. Também verificou-se que os modelos estudados obtiveram bons ajustes aos dados experimentais, com coeficientes de determinação $\left(\mathrm{R}^{2}\right)$ acima de 0,922 e erro relativo $(\mathrm{P})$ abaixo $8,869 \%$ nas diferentes condições experimentais, se destacando os modelos de GAB e Peleg que obtiveram os melhores coeficientes de determinação e os mais baixos valores de erro relativo.

\section{INTRODUÇÃO}

A produção de sementes, o qual possui um processo complexo e uma série de fatores específicos, tem-se destacado na região sul do Brasil a partir de pastagens naturais, sendo um importante recurso utilizado para sustentar a produção pecuária. Aproximadamente $77 \%$ dos animais alimentam-se através da forragem produzida em formações de vegetais (Maia et al., 2006).

As sementes forrageiras constituem-se como a principal fonte de alimento para a pecuária, sendo que as pastagens naturais produzidas a partir dessas sementes fornecem os nutrientes necessários para o crescimento e desenvolvimento para a maior parte dos ruminantes (Almeida et al., 2004). No entanto, o sul do Brasil apresenta outonos e invernos muito rigorosos. Esse fato faz com que nessas épocas ocorra um período de carência alimentar dos rebanhos devido às baixas produções de pastagens, uma vez que os solos são formados principalmente por espécies de crescimento estival (Silveira et al., 1997). Devido a isto, uma atenção tem sido voltada para espécies e/ou cultivares de gramíneas forrageiras perenes que atendam as condições climáticas necessárias para uma boa produção no período considerado crítico (Silveira et al., 1997). Neste contexto, a cevadilha vacarina (Bromus auleticus Trinius) tem se tornado de suma importância para pecuária da Região do Pampa do estado do Rio 
Grande do Sul, pois se trata de uma gramínea perene, nativa dos campos sul brasileiro e de alguns países da America Latina. Essa gramínea apresenta grande capacidade de produção durante o período de maior carência alimentar (outono-inverno) e com boa qualidade da forragem, além de ser resistente à seca e persistir na pastagem por até seis anos (Silva e Moraes, 2009).

Mesmo produzindo sementes viáveis em grande quantidade, algumas sementes do Bromus auleticus Trinius acabam não desempenhando função reprodutiva. Isso se deve ao fato de que aproximadamente $40 \%$ das sementes são falhadas considerando os aspectos fisiológicos da produção (Silva e Moraes, 2009). Essa problemática é de suma importância, pois afeta a qualidade do lote de sementes comprometendo sua distribuição.

Uma alternativa para melhorar os aspectos fisiológicos de sementes nativas pode ser a aplicação da secagem artificial no seu beneficiamento. Esta operação unitária, quando aplicada de forma controlada, possibilita uma colheita antecipada que tem como meta um aumento no percentual de sementes pós-colheita e um acréscimo no padrão de qualidade. A colheita antecipada, além de reduzir as perdas no campo ocasionadas por condições climáticas indesejáveis e/ou pelo ataque de roedores, insetos, pássaros e microorganismos, também possibilita aos produtores obterem melhores preços pelos produtos quando a comercialização ocorrer em épocas de entressafra. Permite também a formação de estoques reguladores de mercado e a oferta de produtos de alta qualidade, no caso de grãos pelas características sensoriais e nutricionais e, no caso das sementes, pela alta porcentagem de germinação e vigor (Kanaan et al., 2013).

O processo de secagem a uma temperatura constante faz com que o teor de água da semente sofra uma variação contínua até que sua pressão de vapor se iguale a pressão de vapor do ar circundante. Sendo assim, o conhecimento da relação entre a umidade da semente e a umidade relativa é de fundamental importância, uma vez que ajuda a estabelecer o teor de umidade final da semente. Tal relação pode ser determinada através do estudo das isotermas de equilíbrio, que são definidas como a relação entre a umidade do material e a umidade relativa correspondente a uma dada temperatura (Moraes et al., 2005).

De acordo com o que foi exposto, o presente trabalho teve como objetivo estudar as isotermas de dessorção de sementes de cevadilha vacariana (Bromus auleticus Trinius) nas temperaturas de 40,50 e $60^{\circ} \mathrm{C}$. A justificativa para o presente estudo está na aplicação das informações obtidas neste trabalho em um futuro estudo sobre o processo de secagem dessas sementes em secador do tipo leito fixo.

\section{MATERIAIS E MÉTODOS}

Este trabalho foi conduzido no Laboratório de Engenharia Química da Universidade Federal do Pampa. Para realização do presente estudo foram utilizadas sementes de Cevadilha vacariana (Bromus auleticus Trinius), obtidas junto a Empresa Brasileira de Pesquisa Agropecuária, Embrapa Pecuária Sul localizada na cidade de Bagé - RS. As sementes de cevadilha vacariana, provenientes da safra 2013, foram colhidas de uma só vez com o intuito de ter uma matéria-prima de mesmo lote e com as mesmas características físicas e fisiológicas iniciais. 
As isotermas de dessorção foram determinadas através do método gravimétrico estático, utilizando-se soluções de ácido sulfúrico em onze diferentes concentrações que garantiram a variação da umidade relativa de 5 a $89 \%$. Os experimentos foram conduzidos nas temperaturas de 40,50 e $60{ }^{\circ} \mathrm{C}$. Cada análise foi realizada em triplicata, utilizando-se recipientes de vidros hermeticamente fechados, suporte e cadinho de plástico. No interior de cada recipiente foram inseridos $30 \mathrm{~mL}$ das soluções de ácido sulfúrico. Também foi inserido em cada cadinho de plástico aproximadamente $1,0 \mathrm{~g}$ de sementes de Cevadilha vacariana (Bromus auleticus Trinius). Os recipientes de vidros hermeticamente fechados foram colocados em estufa de circulação forçada nas temperaturas de 40,50 e $60{ }^{\circ} \mathrm{C}$, sendo realizadas pesagens do cadinho plástico no primeiro, sétimo, décimo e décimo quarto dia de experimento. Essas pesagens foram feitas com o objetivo de verificar o equilíbrio mássico para cada atmosfera estudada. Com o equilíbrio térmico alcançado no décimo quarto dia, determinou-se o teor de umidade das sementes através do método gravimétrico recomendado pela Association of Oficial Analytical Chemists - AOAC, (1997).

Os resultados obtidos nas isotermas de dessorção das sementes de cevadilha vacariana, foram analisados através do ajuste dos dados experimentais aos modelos de BET, GAB, Henderson, Oswin e Peleg, Equações 1 a 5 respectivamente.

$$
\begin{aligned}
& X_{e}=\frac{X_{m} \cdot C \cdot a_{w}}{1-a_{w}}\left[\frac{\left(1-(n+1) \cdot a_{w}{ }^{n}+n \cdot a_{w}^{n+1}\right)}{\left.1-(1-C) \cdot a_{w}-C \cdot a_{w}^{n+1}\right)}\right] \\
& X_{e}=\frac{X_{m} \cdot C_{t} \cdot k \cdot a_{w}}{\left(1-k \cdot a_{w}\right) \cdot\left(1-k \cdot a_{w}+C_{t} \cdot k \cdot a_{w}\right)} \\
& X_{e}=\left[-\frac{\ln \left(1-a_{w}\right)}{a}\right]^{1 / b} \\
& X_{e}=a \cdot\left(\frac{a_{w}}{1-a_{w}}\right)^{b} \\
& X_{e}=k_{1} \cdot a_{w}^{n_{1}}+k_{2} \cdot a_{w}^{n_{2}}
\end{aligned}
$$

O coeficiente de determinação $\left(R^{2}\right)$ e o erro relativo $(P)$, representado na Equação 6, foram os critérios de escolha do melhor modelo para descrever o comportamento da isoterma de dessorção das sementes de cevadilha vacariana. Nessa perspectiva, para um bom ajuste matemático dos modelos, $P$ deve ser menor que $10 \%$ e $\mathrm{R}^{2}$ próximo à unidade.

$$
P=\frac{100}{n} \sum_{i=1}^{n} \frac{\left|X_{e E x p}-X_{e P r e}\right|}{X_{e E x p}}
$$




\section{RESULTADOS E DISCUSSÃO}

Na Tabela 1 são mostrados os dados da atividade $\left(a_{w}\right)$ (Perry e Chilton, 1983) com suas respectivas umidades de equilíbrio $\left(X_{e}\right)$ experimentais para as diferentes temperaturas. Observa-se na Tabela 1 que a umidade de equilíbrio diminui com a redução da atividade de água, sendo esse fato mais expressivo na maior temperatura de estudo e um fenômeno normal para grande parte dos produtos higroscópicos.

Tabela 1 - Umidade de equilíbrio para a Cevadilha vacariana, em função da Temperatura e da atividade de água

\begin{tabular}{cccccc}
\hline \multicolumn{7}{c}{ Temperatura } \\
\hline \multicolumn{7}{c}{$40^{\circ} \mathrm{C}$} & \multicolumn{2}{c}{$50{ }^{\circ} \mathrm{C}$} & $a_{w}$ & $60{ }^{\circ} \mathrm{C}$ \\
\hline$a_{w}$ & $X_{e}$ & $a_{w}$ & $X_{e}$ & $X_{e}$ \\
0,878 & 0,1614 & 0,888 & 0,1068 & 0,890 & 0,0840 \\
0,824 & 0,1502 & 0,829 & 0,0974 & 0,830 & 0,0753 \\
0,753 & 0,1231 & 0,770 & 0,0918 & 0,776 & 0,0744 \\
0,674 & 0,1087 & 0,681 & 0,0895 & 0,682 & 0,0736 \\
0,574 & 0,0999 & 0,582 & 0,0824 & 0,584 & 0,0667 \\
0,470 & 0,0896 & 0,453 & 0,0751 & 0,488 & 0,0630 \\
0,366 & 0,0787 & 0,350 & 0,0660 & 0,388 & 0,0565 \\
0,267 & 0,0642 & 0,280 & 0,0584 & 0,287 & 0,0468 \\
0,178 & 0,0588 & 0,189 & 0,0500 & 0,199 & 0,0396 \\
0,102 & 0,0501 & 0,110 & 0,0439 & 0,124 & 0,0365 \\
0,049 & 0,0370 & 0,055 & 0,0379 & 0,061 & 0,0236 \\
\hline
\end{tabular}

A Tabela 2 apresenta os valores dos parâmetros calculados a partir do ajuste dos modelos de isoterma de GAB, BET, Oswin, Peleg e Henderson. Também nessa tabela são apresentados os respectivos coeficientes de determinação $\left(R^{2}\right)$ e erro relativo $(P)$ para cada modelo ajustado aos dados experimentais.

Tabela 2 - Valores dos parâmetros calculados a partir do ajuste dos modelos de BET, GAB, Oswin, Peleg e Henderson para as sementes de cevadilha vacariana

\begin{tabular}{ccccc}
\hline Modelo & Parâmetro & Valor a $40{ }^{\circ} \mathrm{C}$ & $R^{2}$ & $P(\%)$ \\
\hline \multirow{3}{*}{ BET } & $X_{m}$ & $0,045 \pm 0,002$ & & \\
& $C$ & $-1,88 \mathrm{E}^{45} \pm 6,30 \mathrm{E}^{44}$ & 0,968 & 7,284 \\
& $n$ & $6,903 \pm 0,595$ & & \\
GAB & $X_{m}$ & $0,060 \pm 0,002$ & & \\
& $C t$ & $42,822 \pm 10,523$ & 0,992 & 2,260 \\
Oswin & $K$ & $0,727 \pm 0,0191$ & & \\
& $a$ & $0,909 \pm 0,001$ & 0,993 & 2,730 \\
\multirow{2}{*}{ Peleg } & $b$ & $0,295 \pm 0,008$ & & \\
& $K_{1}$ & $0,112 \pm 0,007$ & & \\
& $n_{1}$ & $0,368 \pm 0,041$ & 0,992 & 2,589 \\
Henderson & $K_{2}$ & $0,110 \pm 0,014$ & & \\
& $n_{2}$ & $5,149 \pm 1,166$ & & \\
& $a$ & $160,471 \pm 50,200$ & 0,974 & 6,750 \\
\hline
\end{tabular}


Tabela 2 - Continuação

\begin{tabular}{|c|c|c|c|c|}
\hline Modelo & Parâmetro & Valor a $50{ }^{\circ} \mathrm{C}$ & $R^{2}$ & $P(\%)$ \\
\hline \multirow{3}{*}{ BET } & $X_{m}$ & $0,042 \pm 0,001$ & \multirow{3}{*}{0,972} & \multirow{3}{*}{4,746} \\
\hline & $c$ & $-1,13 \mathrm{E}^{44} \pm 4,35 \mathrm{E}^{44}$ & & \\
\hline & $n$ & $4,334 \pm 0,266$ & & \\
\hline \multirow{3}{*}{ GAB } & $X_{m}$ & $0,059 \pm 0,003$ & \multirow{3}{*}{0,982} & \multirow{3}{*}{3,928} \\
\hline & $C t$ & $45,255 \pm 9,371$ & & \\
\hline & $K$ & $0,513 \pm 0,037$ & & \\
\hline \multirow{3}{*}{ Oswin } & $a$ & $0,072 \pm 0,001$ & \multirow{2}{*}{0,971} & \multirow{2}{*}{4,905} \\
\hline & $b$ & $0,203 \pm 0,012$ & & \\
\hline & $K_{1}$ & $0,192 \pm 0,050$ & \multirow{4}{*}{0,989} & \multirow{4}{*}{1,675} \\
\hline \multirow{3}{*}{ Peleg } & $n_{1}$ & $0,103 \pm 0,642$ & & \\
\hline & $K_{2}$ & $0,091 \pm 0,048$ & & \\
\hline & $n_{2}$ & $0,700 \pm 0,407$ & & \\
\hline \multirow{2}{*}{ Henderson } & $a$ & $4382,856 \pm 1260,493$ & \multirow{2}{*}{0,991} & \multirow{2}{*}{2,747} \\
\hline & $b$ & $3,385 \pm 0,114$ & & \\
\hline Modelo & Parâmetro & Valor a $60^{\circ} \mathrm{C}$ & $R^{2}$ & $P(\%)$ \\
\hline \multirow{3}{*}{ BET } & $X_{m}$ & $0,033 \pm 0,923$ & \multirow[t]{3}{*}{0,923} & \multirow[t]{3}{*}{8,398} \\
\hline & $c$ & $9,01 \mathrm{E}^{44} \pm 9,55 \mathrm{E}^{44}$ & & \\
\hline & $n$ & $4,382 \pm 0,509$ & & \\
\hline \multirow{3}{*}{ GAB } & $X_{m}$ & $0,058 \pm 0,005$ & \multirow[t]{3}{*}{0,982} & \multirow[t]{3}{*}{3,773} \\
\hline & $C t$ & $25,729 \pm 3,600$ & & \\
\hline & $K$ & $0,388 \pm 0,061$ & & \\
\hline \multirow{2}{*}{ Oswin } & $a$ & $0,057 \pm 0,002$ & \multirow[t]{2}{*}{0,922} & \multirow[t]{2}{*}{8,869} \\
\hline & $b$ & $0,208 \pm 0,021$ & & \\
\hline \multirow{4}{*}{ Peleg } & $K_{1}$ & $0,084 \pm 0,002$ & \multirow[t]{4}{*}{0,988} & \multirow[t]{4}{*}{3,497} \\
\hline & $n_{1}$ & $0,435 \pm 0,022$ & & \\
\hline & $K_{2}$ & $166,717 \pm 1,37 \mathrm{E}^{6}$ & & \\
\hline & $n_{2}$ & $1,15 \mathrm{E}^{6} \pm 1,43 \mathrm{E}^{11}$ & & \\
\hline \multirow{2}{*}{ Henderson } & $a$ & $6292,432 \pm 3563,222$ & \multirow[t]{2}{*}{0,970} & \multirow[t]{2}{*}{5,475} \\
\hline & $b$ & $3,233 \pm 0,206$ & & \\
\hline
\end{tabular}

De acordo com os valores vistos na Tabela 2, todos os modelos testados representam satisfatoriamente as isotermas de dessorção das sementes de cevadilha vacariana (Bromus auleticus Trinius), uma vez que todos os modelos obtiveram valores do coeficiente de determinação $\left(\mathrm{R}^{2}\right)$ maiores que 0,92 e erro relativo $(P)$ abaixo $10 \%$. No entanto os modelos que tiveram os valores ajustados mais constantes para as temperaturas estudadas foram os modelos de GAB com coeficientes de determinação entre 0,982 e 0,992 e erro relativo entre 2,260 e 3,928 e Peleg com 0,988 e 0,992 de coeficientes de determinação e 1,765 e 3,497 de erro relativo. A partir desses valores o modelo escolhido foi o de GAB, por ser uma Equação triparamétrica de polinômio menor que o modelo de Peleg. Além disso, o modelo de GAB permite um bom ajuste dos dados de sorção de produtos até a atividade de água de 0,90 , onde as constantes de modelo $C$ e $K$ são parâmetros de adsorção relacionados com as interações energéticas entre as moléculas da monocamada e as camadas subsequentes num dado sítio de sorção. Esse modelo fornece também os valores de monocamada de água, que indicam a relação com que as reações químicas ocorrem determinando a velocidade de deteriorização biológica através das áreas expostas da matriz sólida. Os valores de umidade da monocamada $\left(X_{m}\right)$ do presente trabalho encontram-se coerentes com estudos de Al-Muhtaseb et al., (2004) e Mclaughlin e Magee (1998), que também relataram a diminuição dos valores da 
monocamada com o aumento da temperatura, o qual afirmam essa tendência devido a variação do estado de agitação e atração entre as moléculas de água com a variação da temperatura.

Na Figura 1 são apresentadas as curvas experimentais das isotermas de dessorção das sementes de cevadilha vacariana (Bromus auleticus Trinius) nas temperaturas de 40, $50 \mathrm{e}$ $60{ }^{\circ} \mathrm{C}$ ajustadas ao modelo de GAB.

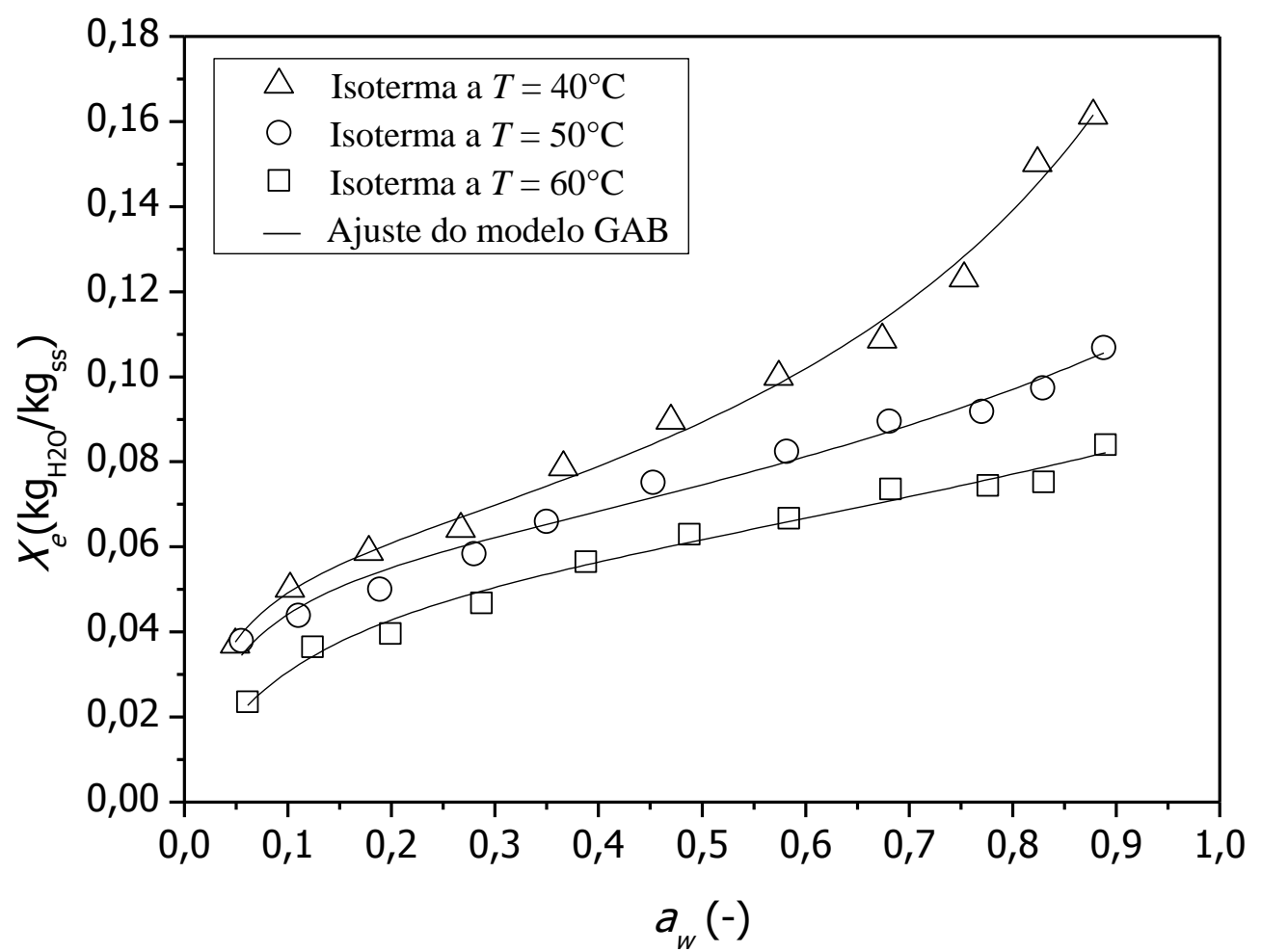

Figura 1 - Isotermas de dessorção das sementes de cevadilha (Bromus Auleticus Trinius) ajustadas pelo modelo de GAB.

Verifica-se na Figura 1 que para um mesmo valor atividade de água o aumento da temperatura promove redução do teor de água de equilíbrio, seguindo a tendência da maioria dos produtos agrícolas. Este comportamento é, geralmente, atribuído a uma redução no número de sítios ativos devido a mudanças químicas e físicas provocada pela variação da temperatura. Além disso, nota-se que as curvas obtidas para as temperaturas de estudo apresentam um formato sigmoidal que é característico de curvas de isotermas do tipo II, segundo a classificação de Brunauer et al., (1938). Essa classificação, que é característica de produtos agrícolas, também foi obtida em trabalhos literários de Kannan et al., (2013) e Almeida et al., (1999).

\section{CONCLUSÃO}

A partir do estudo de isotermas de dessorção para as sementes de cevadilha vacariana (Bromus auleticus Trinius) foi possível prescrever que todos os modelos estudados foram satisfatórios aos dados experimentais, podendo ser utilizados para o cálculo da umidade de equilíbrio higroscópico desse tipo de sementes. Apesar disso, o modelo matemático que 
melhor representou as isotermas nas temperaturas de 40,50 e $60{ }^{\circ} \mathrm{C}$ foi o modelo de $\mathrm{GAB}$ com um coeficiente de determinação acima de 0,982 e o erro relativo abaixo de 3,928. Também foi possível observar que a sementes estudadas apresentaram comportamento do tipo II, ou seja, um formato sigmoidal que é característico para produtos agrícolas.

\section{NOMENCLATURA}

$\begin{array}{ccc}\text { Símbolo } & \text { Notação } & \text { Unidade } \\ a, b, C, C_{t}, k, k_{1}, & \text { Constante de modelo } & {[-]} \\ k_{2}, n, n_{1}, n_{2} & \text { Atividade da água } & {[-]} \\ a_{w} & \text { Números de observações experimentais } & {[-]} \\ n & \text { Umidade de equilíbrio } & {\left[\mathrm{kg}^{\left.-\mathrm{kg}^{-1}\right]}\right.} \\ X_{e} & \text { Umidade de equilíbrio teórico } & {\left[\mathrm{kg} \cdot \mathrm{kg}^{-1}\right]} \\ X_{e P r e} & \text { Umidade de equilíbrio experimental } & {\left[\mathrm{kg} \cdot \mathrm{kg}^{-1}\right]} \\ X_{e \text { Exp }} & \text { Umidade de monocamada molecular } & {[\mathrm{kg} \cdot \mathrm{kg}-1]}\end{array}$

\section{REFERÊNCIAS}

ALMEIDA, F. C.; CECATO, U.; JOBIM, C. C.; CANTO, M. W. Pastagens para Produção de Leite. (Tese de Doutorado). Programa de Pós-graduação em Zootecnia da UEM, 2004.

ALMEIDA, F. DE A. C.; FONSECA, K. S.; GOUVEIA, J. P. G. Secagem natural de gergelim e determinação da umidade de equilíbrio. Revista Brasileira de Engenharia Agrícola e Ambiental, v.3, n.3, p.343-348, 1999.

AL-MUHTASEB, A. H.; McMINN, W. A. M.; MAGEE, T. R. A. Water sorption isotherms of starch powders Part 1: mathematical description of experimental data. Journal of Food Engineering, v.13, n.61, p.297-307, 2004.

ASSOCIATION OF OFFICIAL ANALYTICAL CHEMISTS - AOAC. Official methods of analysis. 16. ed. Washington, D.C., 1995.

BRUNAUER, S.; EMMETT, P. H.; TELLER, E. Adsorption of gases in multimolecular layer. Journal of American Chemistry Society, v.60, n.1, p. 309-312, 1938.

KANNAN, A. F.; FlORES, C. G.; OLIVEIRA, J. C. P.; ALMEIDA, A. R. F. Estudo do processo de reumidificação e das isotermas de dessorção das sementes cornichão (Lotus corniculátus L.). Trabalho apresentado nos anais do XXXVI Congresso Brasileiro de Sistemas Particulados. Maceio - AL, 2013.

LEHN, D. N.; PINTO, L. A. A. Isotermas de equilíbrio e curvas de secagem para arroz em casca em silos de armazenagem. Engenharia na Agricultura, v.12, n.3, p.177-191, 2004.

MAIA, M. S.; SILVA G. M.; PESKE, S. T.; MORAES, C. C.; MEDEIROS, R. B. Produção de sementes de cevadilha-vacariana (bromus auletius trinius) em função da cronologia de desenvolvimento dos perfilhos reprodutivos. Revista Brasileira de Sementes, v.28, n.3, p.13-20, 2006. 
McLAughlin, C. P.; MAGEE, T. R. A. The determination of sorption isotherm and isosteric heats of sorption for potatoes. Journal of Food Engineering, v.13 n.35, p.267280, 1998.

MORAES, M. A.; ROSA, G. S.; PINTO, L. A. A. Estudo das isotermas de equilíbrio para quitina: determinação do calor de dessorção. Trabalho apresentado nos anais do VI Congresso Brasileiro de Engenharia Química em Iniciação Científica. Campians - SP, 2005 (publicado em CD-ROM).

PERRY, R. H.; CHINTON, C. H. Chemical Engineer's Handbook, Editora McGraw-Hill, $5^{\text {a }}$ edição, 1983.

SILVA M. M.; GOUVEIA J. P. G.; ALMEIDA F. DE A. C. Dessorção e calor Isostérico em polpa de manga. Revista Brasileira Agrícola e Ambiental, v.6, n.1, p.123-127, 2002.

SILVA, G. M.; MORAES, C. C. Cevadilha Vacariana (Bromus auleticus Trinius): Histórico, Utilização e Perspectivas. Bagé, RS: Embrapa Pecuária Sul, 2009.

SILVEIRA, L. M.; OLIVEIRA, J. C. P.; MORAES, C. C.; MARQUES, D. G. Análise da diversidade genética em acessos de Bromus auleticus TRINIUS. Revista Ciência Rural, v.27, n.3, p.381-385, 1997.

\section{AGRADECIMENTOS}

Os autores deste trabalho agradecem a UNIPAMPA/Bagé e a EMBRAPA/Pecuária Sul pela infraestrutura e matéria prima disponibilizada e a CAPES pela concessão da bolsa. 